\title{
Wogonin, a flavonoid isolated from Scutellaria baicalensis, has anti-viral activities against influenza infection via modulation of AMPK pathways
}

\author{
R.-K. SEONG, J.-A. KIM, O. S. SHIN
}

Department of Biomedical Sciences, College of Medicine, Korea University Guro Hospital, Seoul, Republic of Korea

Received February 13, 2017; revised May 3, 2017; accepted January 22, 2018

\begin{abstract}
Summary. - Wogonin, a flavonoid isolated from Scutellaria baicalensis, has attracted increasing scientific attention in recent years because of its potent anti-tumor activity. Its role during viral infection has largely been unexplored. Wogonin treatment effectively suppressed both influenza A and B virus replication in Madin-Darby Canine Kidney (MDCK) cells and human lung epithelial (A549) cells. In contrast, wogonin treatment following influenza A virus infection led to up-regulation of interferon (IFN)-induced antiviral signaling. Additionally, influenza A virus infection in A549 cells induced 5' adenosine monophosphate-activated protein kinase (AMPK) phosphorylation and activation in a time-dependent manner and wogonin treatment led to the suppression of AMPK phosphorylation. Furthermore, the treatment with AMPK-specific inhibitor (compound C; CC) attenuated influenza A virus replication. These data suggest that wogonin possesses a potent anti-influenza activity mediated by regulation of AMPK activation, suggesting that wogonin has the potential to be developed as an anti-influenza drug.
\end{abstract}

Keywords: wogonin; influenza; interferon; AMPK; anti-viral signaling

\section{Introduction}

Wogonin (5,7-dihydroxy-8-methoxyflavone) is a flavonoid compound extracted from the roots of Scutellaria baicalensis. Wogonin induces apoptosis in several types of tumor cells, including lung, breast, and glioma cells (Chen et al., 2008; Yu and Kim, 2011; Tsai et al., 2012). For millennia, it has been widely used in medical practice in Asia, mainly for inflammatory and liver diseases, with an excellent safety record; however, the mechanisms underlying its antiviral effects are not well understood.

Influenza virus belongs to the Orthomyxoviridae family and is an important cause of respiratory infection worldwide despite the availability of multiple seasonal influenza vaccines. Although vaccines are the most effective way for

*Corresponding author. E-mail: oshin@korea.ac.kr; phone: +822-2626-3280.

Abbreviations: AMPK $=5$ adenosine monophosphate-activated protein kinase; IFN = interferon; ISGs = interferon-stimulated genes; $\mathrm{MDCK}=$ Madin-Darby Canine Kidney cells; $\mathrm{MxA}=$ myxovirus resistance gene $\mathrm{A} ; \mathrm{NP}=$ nucleoprotein, $\mathrm{NS1}$ = nonstructural protein 1 ; OAS $=2-5^{\prime}$ oligoadenylate synthetase the control and prevention of influenza virus infection, rapid mutation of viral surface glycoproteins hemagglutinin (HA) and neuraminidase (NA) contributes to frequent antigenic changes, which makes annual influenza vaccine strain selection difficult to match with circulating viruses. The influenza virus has vast genetic diversity, comprising 18 HA subtypes and 11 NA subtypes (Garcia-Sastre, 2006) and can infect a wide range of species as diverse as poultry, marine, pigs, horses and humans. Current anti-influenza drugs can be classified into two groups; matrix protein 2 (M2) and neuraminidase inhibitors. Anti-influenza drugs amantadine and rimantadine are M2 ion-channel inhibitors, whereas oseltamivir and zanamivir are neuraminidase inhibitors (Das, 2012). Accumulating evidence suggests that resistance to M2 ion-channel inhibitors and neuraminidase inhibitors has emerged rapidly in recent years (Hurt et al., 2009a,b,c,d, 2011; Speers et al., 2010). Therefore, novel target molecules and/or pathways that can be exploited to reduce viral replication are needed to limit the incidence of influenza infections.

Recent studies have focused on the anti-influenza activities of Scutellaria baicalensis-derived compounds and 
derivatives. In particular, the anti-influenza activities of baicalein and baicalin, components of the roots of Scutellaria baicalensis, have been reported by several groups (Chen et al., 2011; Hour et al., 2013; Sithisarn et al., 2013; Chung et al., 2014; Nayak et al., 2014). Baicalin exerts its anti-influenza virus activity by modulating viral non-structural protein 1 (NS1), which results in the up-regulation of interferon (IFN)induced antiviral signaling and reduced phosphoinositide 3-kinase/Akt (PI3K/Akt) signaling in A549 cells (Nayak et al., 2014). Moreover, baicalein and ribavirin exert a synergistic protective effect against influenza virus pathogenesis in vivo, resulting in an increased survival rate in mice treated with the baicalein/ribavirin combination and a lethal dose of $\mathrm{H} 1 \mathrm{~N} 1$ influenza virus, in comparison with ribavirin alone (Chen et al., 2011). However, the anti-viral effect of wogonin, another plant extract of Scutellaria baicalensis, remains to be determined.

In this study, we measured the antiviral activity of wogonin against influenza A and B virus. Our data suggest that wogonin exerts anti-viral effects by modulating the type I IFN signaling pathway and adenosine monophosphate-activated protein kinase (AMPK) pathway. Additionally, similar to wogonin, AMPK-specific inhibitor, compound C, could serve as an antiviral therapeutic compound during influenza infection. Thus, similar to baicalein and baicalin, wogonin can be considered a potential anti-influenza therapeutic drug.

\section{Materials and Methods}

Reagents and cells. Wogonin was purchased from AdipoGen (San Diego, CA, USA), dissolved in dimethyl sulfoxide (DMSO), and stored at $-20^{\circ} \mathrm{C}$. Human lung epithelial cells (A549) and MadinDarby canine kidney (MDCK) cells were obtained from the American Type Culture Collection (ATCC, Manassas, VA, USA). A549 cells were cultured in RPMI 1640 medium supplemented with $10 \%$ fetal bovine serum (FBS), $100 \mathrm{U} / \mathrm{ml}$ penicillin, and $100 \mu \mathrm{g} / \mathrm{ml}$ streptomycin and maintained at $37^{\circ} \mathrm{C}$ with in a humidified atmosphere with $5 \% \mathrm{CO}_{2}$. MDCK cells were grown in Dulbecco's Modified Eagle's Medium (DMEM) supplemented with 10\% FBS, 100 U/ $\mathrm{ml}$ penicillin, and $100 \mu \mathrm{g} / \mathrm{ml}$ streptomycin. AMPK inhibitor compound $\mathrm{C}$ was purchased from Calbiochem. AMPK activator 5-aminoimidazole-4-carboxamide ribonucleotide (AICAR) was purchased from Sigma-Aldrich (St. Louis, MO, USA).

MTT assay. To evaluate the cytotoxicity of wogonin, MTT assay was performed to determine the cell viability. A549 cells were seeded at density of 20,000 cells $/ \mathrm{ml}$ in 96 -well plates. After $24 \mathrm{~h}$, wogonin $(1,5,10 \mu \mathrm{g} / \mathrm{ml})$ or $\operatorname{AICAR}(0.1,0.25,0.5,1 \mu \mathrm{mol} / \mathrm{l})$ or CC $(1,5,10,20 \mu \mathrm{mol} / \mathrm{l})$ was added, and the incubation was continued for $24 \mathrm{~h}$ at $37^{\circ} \mathrm{C}$. Drugs were added in $2 \%$ serum medium. After the incubation, $0.5 \%$ 3-(4, 5-dimethylthiazolyl-2)-2, 5-diphenyltetrazolium bromide (MTT) (Sigma-Aldrich) solution was added for $4 \mathrm{~h}$, after which DMSO $(100 \mu \mathrm{l} /$ well $)$ was added at room temperature to dissolve the precipitate completely. The optical density was then measured at $570 \mathrm{~nm}$ using a microplate spectrophotometer (Epoch, BioTeK).

Virus plaque assay. Human influenza virus A/Puerto-Rico/8/34 (H1N1) PR8 was provided by Dr. Adolfo Garcia-Sastre (Icahn School of Medicine at Mount Sinai, USA). Clinical influenza strains, such as seasonal H1N1, H3N2 and B (yamagata lineage) were obtained from Korea Bank for Pathogenic Viruses (KBPV). The virus infectivity titers of supernatants were determined by plaque assays (Kim et al., 2016). Viral supernatants diluted in DMEM were added to MDCK cells in 6-well plates. After 2 hours of attachment, viral supernatants were removed and cells were overlaid with Eagle's minimum essential medium (EMEM) (without phenol red, with l-glutamine) (Lonza), 1.5\% LE agarose (Lonza), and $2 \mu \mathrm{g} / \mathrm{ml}$ tolylsulfonyl phenylalanyl chloromethyl ketone (TPCK)-trypsin (Sigma-Aldrich), after which the cells were incubated for 3 days. After the incubation, the infected cells were fixed with $4 \%$ formaldehyde (Sigma-Aldrich) in PBS and stained with $0.5 \%$ crystal violet solution. Plaque-forming units (PFU) were calculated.

Quantitative real time RT-PCR. RNA isolation and cDNA synthesis were conducted by previously described methods (Seong et al., 2016). Briefly, total RNA (0.5-1 $\mu$ g, isolated with Trizol reagent [Invitrogen]) was reverse transcribed to generate cDNA using the RT system (Promega) for $1 \mathrm{~h}$ at $42^{\circ} \mathrm{C}$. The resulting cDNA was used as the template for real-time PCR quantification. Using a SYBR Green PCR Master Mix (Thermo Fisher Scientific), mRNA expression levels of human and influenza genes were measured. The cycling parameters were as follows: $95^{\circ} \mathrm{C}$ for $15 \mathrm{~min}$, followed by 40 cycles of $30 \mathrm{~s}$ at $95^{\circ} \mathrm{C}$ and $1 \mathrm{~min}$ at $60^{\circ} \mathrm{C}$. Glyceraldehyde 3-phosphate dehydrogenase (GAPDH) mRNA was used as a normalizing control. Calculations of expression levels were normalized using the $\Delta C \mathrm{t}$ method, wherein the amount of target, normalized to an endogenous reference and relative to a calibrator, is given by $2^{-\Delta \Delta} \mathrm{Ct}$, where $\mathrm{Ct}$ is the cycle number of the detection threshold.

Western blotting. Cells were lysed at the specified times postinfection with RIPA buffer (Sigma-Aldrich). Lysates were resolved by sodium dodecyl sulfate polyacrylamide gel electrophoresis (SDS-PAGE) on 8 12\% acrylamide gels. Proteins were transferred to polyvinylidene difluoride membranes (Merck), and blocked with $5 \%(\mathrm{w} / \mathrm{v})$ skim milk in Tris-buffered saline $(0.2 \mathrm{~mol} / \mathrm{l}$ Tris, $1.36 \mathrm{~mol} / \mathrm{l}$ $\mathrm{NaCl}$ ) supplemented with $0.1 \%(\mathrm{v} / \mathrm{v})$ Tween-20 (TBS-T) for $1 \mathrm{~h}$ at room temperature. This was followed by overnight incubation with primary antibodies against phospho-AMPK, total AMPK, phospho-ACC, total-ACC, beclin-1, LC3, phospho-IRF3, totalIRF3, phospho-AKT, total AKT, caspase-3, PARP (Cell Signaling). Anti- $\beta$-actin (Abgent) and anti-GAPDH (Sigma-Aldrich) antibodies were used as loading controls. For influenza A virus protein expression, anti-NS1 antibodies (Santa Cruz Biotechnology), and anti-NP antibodies (Sino biological) were used. After 3 washes in TBS/T, the membranes were incubated with HRP-conjugated anti-rabbit or anti-mouse IgG secondary antibodies for $1 \mathrm{~h}$ at $25^{\circ} \mathrm{C}$. Membranes were washed with TBS-T, incubated with Western Lumi Pico solution (ECL solution kit) (DoGen), and exposed to a film. 
Statistical analysis. Data were analyzed using Student's $t$-test to determine the significance of differences between the means of 2 groups. Statistics were performed using Prism software. $P$-values $<0.05$ were considered significant.

\section{Results}

Wogonin shows potent anti-influenza activity in vitro

To evaluate the effect of wogonin (chemical structure shown in Fig. 1a) on cell viability, the MTT assay was performed. Briefly, A549 cells were treated with increasing concentrations of wogonin $(1,5$, and $10 \mu \mathrm{g} / \mathrm{ml})$ and incubated for $24 \mathrm{~h}$. Cell viability was measured with respect to vehicle-treated (DMSO) controls (w0) by the MTT assay. As shown in Fig. 1b, wogonin treatment did not cause cytotoxicity in A549 cells.

Plaque assay results indicated that the inhibitory concentration by $50 \%\left(\mathrm{IC}_{50}\right)$ of wogonin against the PR8 virus was $10 \mu \mathrm{g} / \mathrm{ml}$ (data not shown). At 16 hours post infection (hpi) with PR8 (MOI = 0.1), nucleoprotein (NP), and nonstructural protein 1 (NS1) influenza protein levels were significantly reduced in lysates from cells treated with $10 \mu \mathrm{g} / \mathrm{ml}$ wogonin (Fig. 1c). Additional plaque assays were performed to determine whether wogonin suppressed viral replication of other influenza strains, including A/H1N1, A/H3N2 and $\mathrm{B} /$ yamagata. The anti-viral effect of wogonin against both $A$ and $B$ influenza strains was significantly enhanced than that of baicalein, resulting in the inhibition of viral replication (Fig. 1d).

Wogonin induces IFN response during influenza infection. Transcript levels of type I IFN (IFN- $\beta)$, type III IFN (IFN- $\lambda 1)$, and interferon-stimulated genes (ISGs) (myxovirus resistance gene A $[M x A], 2-5$ ' oligoadenylate synthetase $[O A S]$ were measured to determine whether wogonin treatment modulated the host anti-viral immune response. Cells exposed to $10 \mu \mathrm{g} / \mathrm{ml}$ wogonin had significantly increased transcript levels of IFN- $\beta$ and IFN- $\lambda$ in comparison with those of control-treated cells and cells treated with lower concentrations of wogonin (Fig. 2). Additionally, transcript levels of IFN downstream molecules $M x A$ and $O A S$ were significantly up-regulated following treatment with $10 \mu \mathrm{g} / \mathrm{ml}$ wogonin.

Influenza A virus infection in A549 cells resulted in AMPK activation

To investigate signaling pathways modulated by wogonin upon influenza A virus infection, A549 cells were infected (a)<smiles>COc1c(O)cc(O)c2c(=O)cc(-c3ccccc3)oc12</smiles>

(c)

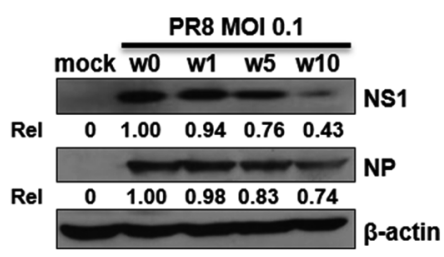

(b)

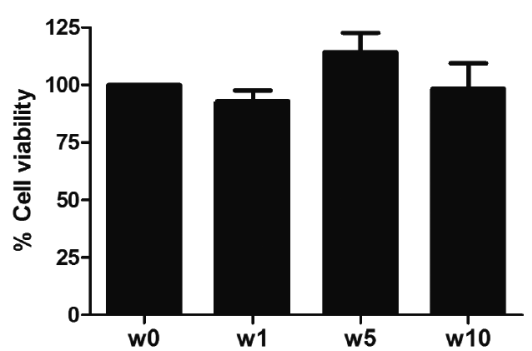

(d)

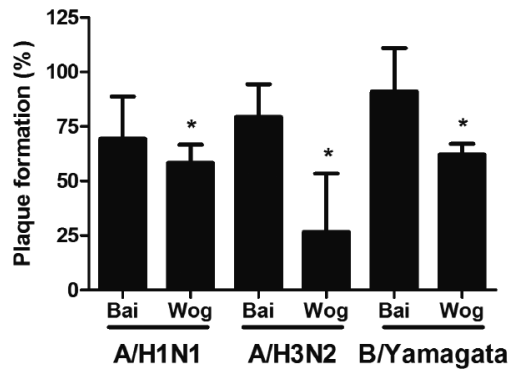

Fig. 1

Viral replication efficiency in wogonin-treated cells

(a) The chemical structure of wogonin isolated from Scutellaria baicalensis is shown. (b) A549 cells were treated with various concentrations (1, 5, and $10 \mu \mathrm{g} / \mathrm{ml}$ ) of wogonin (w) for $24 \mathrm{~h}$, after which cell viability was measured using the MTT assay. Data are presented as a percentage (\%) of the cell viability of the control DMSO-treated cells (100\%). The average of 3 independent experiments is shown. (c) Western blotting was performed for viral NP, and NS1 proteins using total cell lysates. Levels of cellular actin are shown as loading controls. Results are representative of 3 independent experiments. (d) Plaque assays were performed with the influenza A (H1N1, H3N2) and B (Yamagata lineage) strains of influenza virus. After $72 \mathrm{~h}$, the number of plaques was counted. The results are presented as the percentage decrease with respect to control-treated cells $(100 \%)$. ${ }^{\star} p<0.05$ vs. the control-treated group. 

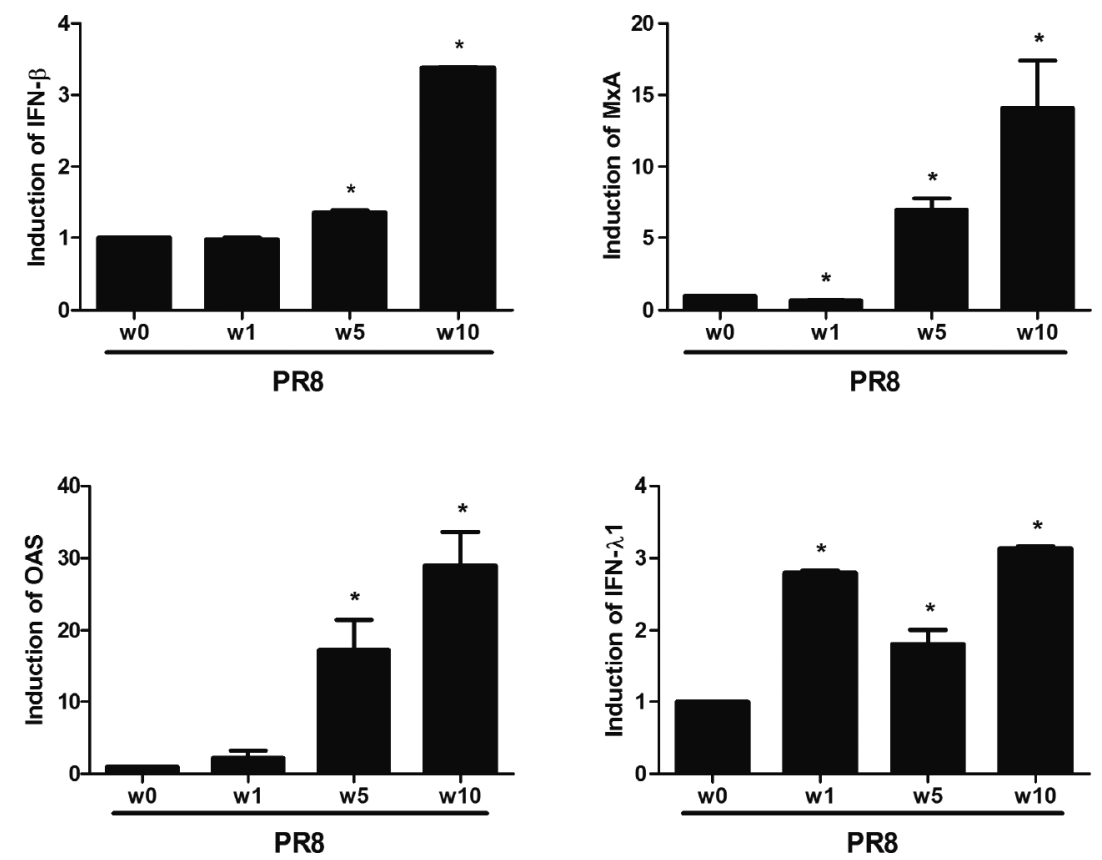

Fig. 2

Effect of wogonin on host interferon-induced signaling and gene expression in influenza-infected A549 cells

Changes in transcriptional expression of $I F N-\beta(A), M x A(B), O A S(C)$, and $I F N-\lambda 1(D)$ in A549 cells infected with the H1N1-PR8 virus $(M O I=0.1)$ in the presence or absence of wogonin $(\mathrm{w})$ at various concentrations were measured by real-time PCR. Transcript expression levels were calculated in relation to the expression level of GAPDH and expressed as a fold induction in comparison with the expression level of mock-treated cells. Statistical analysis: ${ }^{*} p<0.05$ vs. the control-treated group (w0).

with PR8 virus at an MOI of 0.1 and protein samples were collected $0,4,8,16$, and $24 \mathrm{~h}$ after infection. Protein expression levels of NS1, and NP began to increase 8 hpi and were prominently expressed until $24 \mathrm{hpi}$. It is well established that autophagosome formation is induced by influenza A virus infection (Zhou et al., 2009). Similarly, our data indicate that influenza infection in A549 cells increased protein levels of autophagy-associated proteins, such as beclin-1 and LC3-II (Fig. 3a)

We tested whether influenza A virus infection would lead an increase in the activation of AMPK. It was shown that phosphorylation of AMPK began at 4 hpi and continued until 16 hpi. To determine the role of AMPK during influenza infection, cells were infected with the influenza virus and treated with wogonin, after which AMPK-a phosphorylation (Thr172) was measured by western blotting. Phosphorylation of AMPK-a Thr172 was dramatically suppressed following wogonin treatment (Fig. 3b). In addition, the levels of beclin-1 were inhibited slightly by higher dose of wogonin treatment, suggesting that AMPK pathway could be linked to the regulation of autophagy.

Furthermore, we assessed the effect of wogonin on IFN-induced signaling. Protein levels of phosphorylated interferon regulatory factor-3 (IRF-3) and p-AKT were measured. The phosphorylation of IRF-3 was suppressed by influenza infection, whereas wogonin treatment increased phospho-IRF-3 expression in comparison with that of DMSO control-treated (w0) cells. Furthermore, as shown in Fig. 3b, wogonin inhibited influenza virus-induced Akt phosphorylation at a concentration of $10 \mu \mathrm{g} / \mathrm{ml}$. Levels of total Akt remained constant, indicating that the observed changes in Akt phosphorylation were not due to changes in cellular protein levels. To determine whether wogonin would affect apoptosis of influenza-infected cells, we also measured activated caspase- 3 and cleaved PARP by western blotting. As shown in Fig. 3b, wogonin treatment resulted in an increase in cleaved PARP and activated caspase- 3 expression in A549 cells, suggesting that wogonin treatment can induce apoptosis.

\section{Pharmacological inhibition of AMPK via compound C} decreased viral yield

To evaluate the effect of AMPK-modulating drugs on cell viability, MTT assays were performed. Briefly, A549 cells were treated with increasing concentrations of 5-ami- 

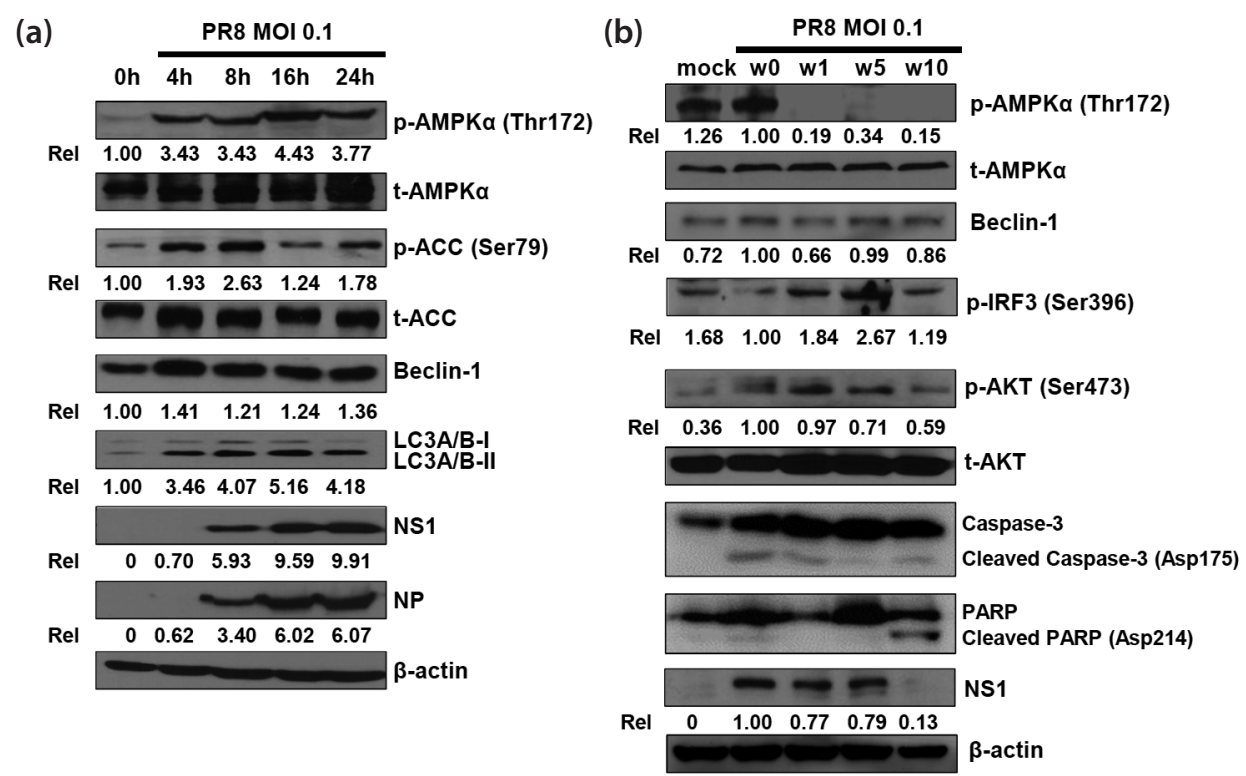

Fig. 3

Wogonin's effect on host cellular signaling pathways followed by influenza infection

(a) A549 cells were mock-infected (m) or infected with the PR8 virus at a multiplicity of infection (MOI) of 0.1. Cells were harvested at 0, 4, 8, 16, and 24 hpi and analyzed by western blotting with antibodies specific for phospho-AMPK- $\alpha$ /total AMPK-a, phospho ACC/total ACC, beclin-1, and LC3. Influenza protein expressions were measured using anti-NS1, and anti-NP antibodies. The ratio of phosphorylated protein expression relative to total protein expression was determined using densitometry. (b) A549 cells were either mock infected or infected with the PR8 virus at MOI of 0.1 in the presence and absence of wogonin $(0,1,5$, and $10 \mu \mathrm{g} / \mathrm{ml})$. The samples were collected 24 hours post infection. The ratio of phospho-AMPK- $\alpha$ expression relative to total AMPK- $\alpha$ expression was determined by western blotting. Relative expression levels of beclin-1, influenza NS1, p-IRF3, p-AKT, activated caspase 3 and cleaved PARP normalized to loading control $\beta$-actin are presented below the blots. The reported results are representative of three independent experiments.

noimidazole-4-carboxamide 1-D-ribofuranoside, AICAR (an AMPK activator) or compound C (an AMPK inhibitor; CC) and incubated for $24 \mathrm{~h}$. As shown in Fig. 4a, AICAR or compound $\mathrm{C}$ produced minimal reductions in cell viability, with the exception of compound $\mathrm{C}$ at $20 \mu \mathrm{mol} / \mathrm{l}$. Thus, we used $10 \mu \mathrm{mol} / \mathrm{l}$ compound $\mathrm{C}$ and $0.5 \mu \mathrm{mol} / \mathrm{l} \mathrm{AICAR}$ in subsequent experiments.

Given that compound C treatment during influenza infection decreased phosphorylation of AMPK- $\alpha$ (Fig. 4c), we tested whether AMPK inhibition via compound $C$ impacted viral replication. Production of viral progeny was assessed in control, compound C-treated, and AICAR-treated MDCK cells by plaque reduction assay. Compound $\mathrm{C}$ significantly reduced the number of plaques in a dose-dependent manner, whereas AICAR treatment had a tendency to increase the number of plaque forming units (PFU) (Fig. 4b). Similar to the observed compound C-mediated suppression of viral titer, autophagy inhibitor 3-MA also reduced viral titer, consistent with previous reports (data not shown) (Zhou et al., 2009). Moreover, NP expression was significantly reduced in cells treated with $10 \mu \mathrm{mol} / \mathrm{l}$ compound $\mathrm{C}$, whereas the abundance of NP was similar in control cells and cells treated with $0.5 \mu \mathrm{mol} / \mathrm{l}$ AICAR (Fig. $4 \mathrm{~d}$ ). These results sug- gest that compound $\mathrm{C}$ affects viral plaque formation, as well as viral gene transcript and protein expression, via AMPK inhibition.

\section{Discussion}

Scutellaria baicalensis exhibits therapeutic effects against wide range of diseases. Six flavones are proven to be the major bioactive flavones in Scutellaria baicalensis, presenting the forms of aglycones (baicalein, wogonin, oroxylin A) and glycosides (baicalin, wogonoside, oroxylin A-7-glucuronide). Wogonin has been suggested to have the therapeutic effects in the treatment of inflammation, cancers and virus-related diseases (Tai et al., 2005). In this study, we investigated the antiviral role of wogonin against influenza virus infection. Previously, it was shown that baicalin, a flavonoid derived from the root of Scutellaria baicalensis, was effective against influenza virus replication (Nayak et al., 2014). The $\mathrm{EC}_{50}$ of baicalin against $\mathrm{H} 1 \mathrm{~N} 1$ and $\mathrm{H} 3 \mathrm{~N} 2$ were found to be $43.3 \mu \mathrm{g} / \mathrm{ml}$ and $104.9 \mu \mathrm{g} / \mathrm{ml}$, respectively, whereas our data identified $\mathrm{IC}_{50}$ of wogonin against the $\mathrm{H} 1 \mathrm{~N} 1$ virus as low as $10 \mu \mathrm{g} / \mathrm{ml}$. Additionally, when compared with baicalein, wogonin's an- 
(a)

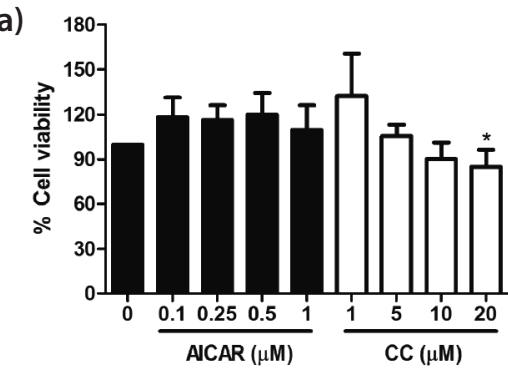

(c)

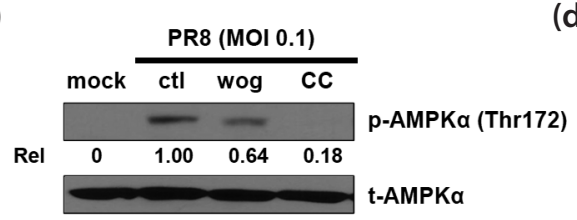

(b)

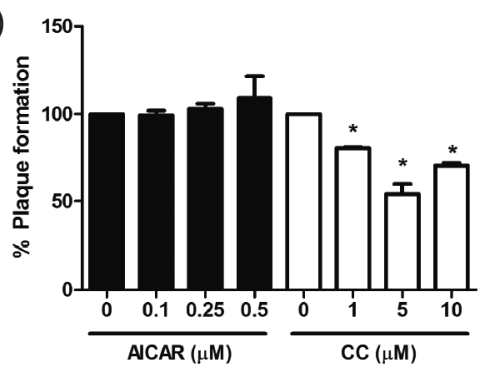

(d)

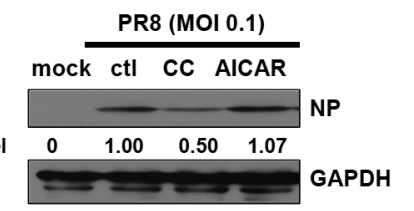

Fig. 4

AMPK inhibitor, compound $\mathrm{C}$, has a suppressive effect on plaque formation

A549 cells were treated with the indicated concentrations of AMPK inhibitor compound C (CC) and AMPK activator AICAR. (a) Cell viability was measured using the MTT assay. Data are presented as a percentage (\%) of the cell viability of control cells. The average of 3 independent experiments is shown. (b) Plaque assays were performed to determine viral progeny formation followed by the treatment with various concentrations of CC and AICAR. After $72 \mathrm{~h}$, the numbers of plaques were counted. Data are presented as the percentage (\%) decrease in the number of plaque forming units with respect to that of the control-treated cells, which was normalized to $100 \%$. Three independent experiments were performed. The average of all experiments is shown. ${ }^{*} p<0.05$ vs. the DMSO control-treated group. (c) Phosphorylation of AMPK in the presence of control DMSO (ctl), $10 \mu \mathrm{g} / \mathrm{ml}$ wogonin (wog), or $10 \mu \mathrm{mol} / \mathrm{l} \mathrm{CC}$ was measured by western blotting. (d) Total cell lysates were prepared and western blotting was performed to measure viral NP protein levels. GAPDH was used as a loading control. The results are representative of 3 independent experiments.

tiviral activities against both A and B influenza strains were stronger, given that the plaque formation was inhibited more than $40 \%$ by wogonin. Thus, this suggests that wogonin may provide broader and more robust spectrum of antiviral activities than baicalein.

Studies have shown that wogonin suppresses replication of multiple viruses, such as vesicular stomatitis virus (BlachOlszewska et al., 2008), hepatitis B virus (Guo et al., 2007), varicella zoster virus (Choi et al., 2015) and human papilloma virus (Kim et al., 2013). Recent studies have demonstrated that baicalein inhibits influenza A virus replication in cell culture and in mice, in addition to increasing the antiviral efficacy of ribavirin (Xu et al., 2010; Chung et al., 2014). As shown by Nayak et al. (2014), baicalin's antiviral mode of action was due to disruption of NS1 protein binding to PI3K subunit, which counteracts cellular antiviral responses by downregulating interferon induction and upregulation of PI3K/Akt signaling. Thus, it will be interesting to examine whether wogonin's activity may also depend on the inhibition of NS1 function because wogonin is structurally and potentially functionally related to baicalin.

So far, there are few classes of drugs licensed by the U.S. Food and Drug Administration that are effective against influenza A virus (Vigant et al., 2015). These drugs are M2 channel blockers (amantadine and rimantadine) and RNA polymerase inhibitors (zanamivir and oseltamivir).
The ongoing emergence of anti-influenza drug resistance imposes an urgent need to discover new and cost-effective anti-influenza drugs. Influenza B viruses cause annual outbreaks of respiratory illness in humans and are increasingly recognized as a major cause of influenza-associated morbidity and mortality, although studies on IBVs are lacking (Paul Glezen et al., 2013). Our data suggest that wogonin not only inhibits replication of influenza A virus strains, but it also attenuates the plaque formation of influenza $B$ strains, thus suggesting a broad anti-viral effect on diverse influenza strains.

Influenza virus infection usually results in the inhibition of type I IFN at early timepoints. NS1 is well known antagonist of type I IFN and suppresses the type I IFN response by interacting with RIG-I (Chan and Gack, 2015). We found that wogonin increased antiviral signaling via phosphorylation of IRF3 and activation of interferon-stimulated genes (ISGs), such as $M x A$ and OAS. MxA is upregulated by type I and III mediated antiviral signaling pathways and inhibits influenza virus infection by blocking viral transcription and replication (Haller and Kochs, 2011). In addition to $I F N-\beta$, wogonin also induced IFN- $\lambda$ expression. These data altogether suggest that wogonin's anti-viral effect may depend on the induction of both type I and III IFN.

Another important finding of our study is that wognin's antiviral activities may depend on the inhibition of AMPK, 
which is an important metabolic stress response kinase that regulates both energy-producing catabolic processes and energy-consuming anabolic processes. In addition to AMPK's role as central regulator of both lipid and glucose metabolism, the role of AMPK in infection by various viruses, including human cytomegalovirus (HCMV), varicella zoster virus (VZV), hepatitis C virus (HCV), and Rift Valley fever virus (RVFV), is mediated by effects on ATP-generating and ATP-consuming metabolic pathways ( Mankouri et al., 2010; McArdle et al., 2012; Moser et al., 2012; Choi et al., 2015). For example, during HCMV infection, inhibition of AMPK by compound $C$ produces a metabolic environment unsuitable for viral replication (McArdle et al., 2012). In our data, we found that AMPK phosphorylation is upregulated upon influenza infection and AMPK inhibition results in attenuation of virus replication. Previous reports suggested a regulatory role of AMPK substrate, acetyl CoA carboxylase (ACC), in influenza replication, indicating that the ACC inhibitor TOFA (5-(tetradecyloxy)-2-furoic acid) and fatty acid synthase (FAS) inhibitor C75 inhibited influenza A virus replication (Munger et al., 2008). In accordance with this report, our results with compound $\mathrm{C}$ show a suppressive effect on viral plaque formation, raising the possibility that AMPK pathways can be a target for broadly anti-viral therapeutics. Thus, it will be interesting to investigate whether combinations of AMPK or ACC inhibitor with wogonin produce synergistic anti-viral effects against influenza virus infection.

Given that wogonin has a robust anti-viral function against influenza viruses, it will be interesting to evaluate its role using in vivo animal model. Taken together, the present findings show that wogonin possesses anti-influenza activity via modulation of AMPK pathways.

Acknowledgments. This research was supported by Basic Science Research Program through the National Research Foundation of Korea (NRF) funded by the Ministry of Science, ICT \& Future Planning (NRF-2016R1C1B2006493).

\section{References}

Blach-Olszewska Z, Jatczak B, Rak A, Lorenc M, Gulanowski B, Drobna A, Lamer-Zarawska E (2008): Production of cytokines and stimulation of resistance to viral infection in human leukocytes by Scutellaria baicalensis flavones. J. Interferon Cytokine Res. 28, 571-581. https://doi. org/10.1089/jir.2008.0125

Chan YK, Gack MU (2015): RIG-I-like receptor regulation in virus infection and immunity. Curr. Opin. Virol. 12, 7-14. https://doi.org/10.1016/j.coviro.2015.01.004

Chen L, Dou J, Su Z, Zhou H, Wang H, Zhou W, Guo Q, Zhou C (2011): Synergistic activity of baicalein with ribavirin against influenza A (H1N1) virus infections in cell cul- ture and in mice. Antiviral Res. 91, 314-320. https://doi. org/10.1016/j.antiviral.2011.07.008

Chen LG, Hung LY, Tsai KW, Pan YS, Tsai YD, Li YZ, Liu YW (2008): Wogonin, a bioactive flavonoid in herbal tea, inhibits inflammatory cyclooxygenase- 2 gene expression in human lung epithelial cancer cells. Mol. Nutr. Food Res. 52, 1349-1357. https://doi.org/10.1002/mnfr.200700329

Choi EJ, Lee CH, Kim YC, Shin OS (2015): Wogonin inhibits Varicella-Zoster (shingles) virus replication via modulation of type I interferon signaling and adenosine monophosphate-activated protein kinase activity. J. Funct. Foods 17, 399-409. https://doi.org/10.1016/j.jff.2015.05.031

Chung ST, Chien PY, Huang WH, Yao CW, Lee AR (2014): Synthesis and Anti-influenza Activities of Novel Baicalein Analogs. Chem. Pharm. Bull. (Tokyo) 62, 415-421. https://doi. org/10.1248/cpb.c13-00897

Das K (2012): Antivirals targeting influenza A virus. J. Med. Chem. 55, 6263-6277. https://doi.org/10.1021/jm300455c

Garcia-Sastre A (2006): Antiviral response in pandemic influenza viruses. Emerg. Infect. Dis. 12, 44-47. https://doi. org/10.3201/eid1201.051186

Guo Q, Zhao L, You Q, Yang Y, Gu H, Song G, Lu N, Xin J (2007): Anti-hepatitis B virus activity of wogonin in vitro and in vivo. Antiviral Res. 74, 16-24. https://doi.org/10.1016/j. antiviral.2007.01.002

Haller O, Kochs G (2011): Human MxA protein: an interferoninduced dynamin-like GTPase with broad antiviral activity. J. Interferon Cytokine Res. 31, 79-87. https://doi. org/10.1089/jir.2010.0076

Hour MJ, Huang SH, Chang CY, Lin YK, Wang CY, Chang YS, Lin CW (2013): Baicalein, Ethyl Acetate, and Chloroform Extracts of Scutellaria baicalensis Inhibit the Neuraminidase Activity of Pandemic 2009 H1N1 and Seasonal Influenza A Viruses. Evid. Based Complement Alternat. Med. 2013, 750803. https://doi.org/10.1155/2013/750803

Hurt AC, Deng YM, Ernest J, Caldwell N, Leang L, Iannello P, Komadina N, Shaw R, Smith D, Dwyer DE, Tramontana AR, Lin RT, Freeman K, Kelso A, Barr IG (2011): Oseltamivir-resistant influenza viruses circulating during the first year of the influenza A(H1N1) 2009 pandemic in the Asia-Pacific region, March 2009 to March 2010. Euro Surveill. 16.

Hurt AC, Ernest J, Deng YM, Iannello P, Besselaar TG, Birch C, Buchy P, Chittaganpitch M, Chiu SC, Dwyer D, Guigon A, Harrower B, Kei IP, Kok T, Lin C, McPhie K, Mohd A, Olveda R, Panayotou T, Rawlinson W, Scott L, Smith D, D'Souza H, Komadina N, Shaw R, Kelso A, Barr IG (2009a): Emergence and spread of oseltamivir-resistant A(H1N1) influenza viruses in Oceania, South East Asia and South Africa. Antiviral Res. 83, 90-93. https://doi. org/10.1016/j.antiviral.2009.03.003

Hurt AC, Holien JK, Barr IG (2009b): In vitro generation of neuraminidase inhibitor resistance in $\mathrm{A}(\mathrm{H} 5 \mathrm{~N} 1)$ influenza viruses. Antimicrob. Agents Chemother. 53, 4433-4440. https://doi.org/10.1128/AAC.00334-09

Hurt AC, Holien JK, Parker M, Kelso A, Barr IG (2009c): Zanamivir-resistant influenza viruses with a novel neurami- 
nidase mutation. J. Virol. 83, 10366-10373. https://doi. org/10.1128/JVI.01200-09

Hurt AC, Holien JK, Parker MW, Barr, IG (2009d): Oseltamivir resistance and the $\mathrm{H} 274 \mathrm{Y}$ neuraminidase mutation in seasonal, pandemic and highly pathogenic influenza viruses. Drugs 69, 2523-2531. https://doi.org/10.2165/11531450000000000-00000

Kim JA, Seong RK, Shin, OS (2016): Enhanced Viral Replication by Cellular Replicative Senescence. Immune Netw. 16, 286-295. https://doi.org/10.4110/in.2016.16.5.286

Kim MS, Bak Y, Park YS, Lee DH, Kim JH, Kang JW, Song HH, Oh SR, Yoon do, Y (2013): Wogonin induces apoptosis by suppressing E6 and E7 expressions and activating intrinsic signaling pathways in HPV-16 cervical cancer cells. Cell Biol. Toxicol. 29, 259-272. https://doi.org/10.1007/ $\underline{\text { s10565-013-9251-4 }}$

Mankouri J, Tedbury PR, Gretton S, Hughes ME, Griffin SD, Dallas ML, Green KA, Hardie DG, Peers C, Harris M (2010): Enhanced hepatitis $C$ virus genome replication and lipid accumulation mediated by inhibition of AMP-activated protein kinase. Proc. Natl. Acad. Sci. USA 107, 1154911554. https://doi.org/10.1073/pnas.0912426107

McArdle J, Moorman NJ, Munger J (2012): HCMV targets the metabolic stress response through activation of AMPK whose activity is important for viral replication. PLoS Pathog. 8, e1002502. https://doi.org/10.1371/journal.ppat.1002502

Moser TS, Schieffer D, Cherry S (2012): AMP-activated kinase restricts Rift Valley fever virus infection by inhibiting fatty acid synthesis. PLoS Pathog. 8, e1002661. https:// doi.org/10.1371/journal.ppat.1002661

Munger J, Bennett BD, Parikh A, Feng XJ, McArdle J, Rabitz HA, Shenk T, Rabinowitz JD (2008): Systems-level metabolic flux profiling identifies fatty acid synthesis as a target for antiviral therapy. Nat. Biotechnol. 26, 1179-1186. https:// doi.org/10.1038/nbt.1500

Nayak MK, Agrawal AS, Bose S, Naskar S, Bhowmick R, Chakrabarti S, Sarkar S, Chawla-Sarkar, M (2014): Antiviral activity of baicalin against influenza virus H1N1-pdm09 is due to modulation of NS1-mediated cellular innate immune responses. J. Antimicrob. Chemother. 69, 1298-1310. https://doi.org/10.1093/jac/dkt534
Paul Glezen W, Schmier JK, Kuehn CM, Ryan KJ, Oxford J (2013): The burden of influenza B: a structured literature review. Am. J. Public Health 103, e43-51. https://doi.org/10.2105/ AJPH.2012.301137

Seong RK, Choi YK, Shin OS (2016): MDA7/IL-24 is an antiviral factor that inhibits influenza virus replication. J. Microbiol. 54, 695-700. https://doi.org/10.1007/s12275016-6383-2

Sithisarn P, Michaelis M, Schubert-Zsilavecz M, Cinatl J, Jr. (2013): Differential antiviral and anti-inflammatory mechanisms of the flavonoids biochanin A and baicalein in H5N1 influenza A virus-infected cells. Antiviral Res. 97, 41-48. https://doi.org/10.1016/j.antiviral.2012.10.004

Speers DJ, Williams SH, Pinder M, Moody HR, Hurt AC, Smith, DW (2010): Oseltamivir-resistant pandemic (H1N1) 2009 influenza in a severely ill patient: the first Australian case. Med. J. Aust. 192, 166-168.

Tai MC, Tsang SY, Chang LY, Xue H (2005): Therapeutic potential of wogonin: a naturally occurring flavonoid. CNS Drug Rev. 11, 141-150. https://doi.org/10.1111/j.1527-3458.2005. tb00266.x

Tsai CF, Yeh WL, Huang SM, Tan TW, Lu DY (2012): Wogonin induces reactive oxygen species production and cell apoptosis in human glioma cancer cells. Int. J. Mol. Sci. 13, 9877-9892. https://doi.org/10.3390/ijms13089877

Vigant F, Santos NC, Lee B (2015): Broad-spectrum antivirals against viral fusion. Nat. Rev. Microbiol. 13, 426-437. https://doi.org/10.1038/nrmicro3475

Xu G, Dou J, Zhang L, Guo Q, Zhou C (2010): Inhibitory effects of baicalein on the influenza virus in vivo is determined by baicalin in the serum. Biol. Pharm. Bull. 33, 238-243. https://doi.org/10.1248/bpb.33.238

Yu JS, Kim AK (2011): Wogonin induces apoptosis by activation of ERK and p38 MAPKs signaling pathways and generation of reactive oxygen species in human breast cancer cells. Mol. Cells 31, 327-335. https://doi.org/10.1007/ $\underline{\text { s10059-011-0041-7 }}$

Zhou Z, Jiang X, Liu D, Fan Z, Hu X, Yan J, Wang M, Gao GF (2009): Autophagy is involved in influenza A virus replication. Autophagy 5, 321-328. https://doi.org/10.4161/ auto.5.3.7406 